\title{
Dexmedetomidine alleviates early brain injury following traumatic brain injury by inhibiting autophagy and neuroinflammation through the ROS/Nrf2 signaling pathway
}

\author{
XIAOYAN FENG $^{1 *}$, WEIWEI MA ${ }^{2 *}$, JIE ZHU $^{1}$, WEI JIAO $^{1}$ and YUHAI WANG ${ }^{1}$ \\ ${ }^{1}$ Department of Neurosurgery, Wuxi Clinical College of Anhui Medical University, 904th Hospital of \\ Joint Logistic Support Force of PLA, Wuxi, Jiangsu 214044; ${ }^{2}$ Clinical Medicine Five-Year Program, \\ 11 Class, 2019 Grade, Wannan Medical College, Wuhu, Jiangsu 241002, P.R. China
}

Received April 22, 2021; Accepted June 29, 2021

DOI: $10.3892 / \mathrm{mmr} .2021 .12300$

\begin{abstract}
Traumatic brain injury (TBI) is a major public health problem and a major cause of mortality and disability that imposes a substantial economic burden worldwide. Dexmedetomidine (DEX), a highly selective $\alpha$-2-adrenergic receptor agonist that functions as a sedative and analgesic with minimal respiratory depression, has been reported to alleviate early brain injury (EBI) following traumatic brain injury by reducing reactive oxygen species (ROS) production, apoptosis and autophagy. Autophagy is a programmed cell death mechanism that serves a vital role in neuronal cell death following TBI. However, the precise role of autophagy in DEX-mediated neuroprotection following TBI has not been confirmed. The present study aimed to investigate the neuroprotective effects and potential molecular mechanisms of DEX in TBI-induced EBI by regulating neural autophagy in a C57BL/6 mouse model. Mortality, the neurological score, brain water content, neuroinflammatory cytokine levels, ROS production, malondialdehyde levels and neuronal death were evaluated by TUNEL staining, Evans blue extravasation, ELISA, analysis of ROS/lipid peroxidation and western blotting. The results showed that DEX treatment markedly increased the survival rate and neurological score, increased neuron survival, decreased the expression of the LC3, Beclin-1 and NF- $\mathrm{KB}$ proteins, as well as the cytokines IL-1 $\beta$, IL- 6 and TNF- $\alpha$, which indicated that DEX-mediated inhibition of autophagy and neuroinflammation ameliorated neuronal death following
\end{abstract}

Correspondence to: Dr Yuhai Wang, Department of Neurosurgery, Wuxi Clinical College of Anhui Medical University, 904th Hospital of Joint Logistic Support Force of PLA, 101 Xing Yuan North Road, Wuxi, Jiangsu 214044, P.R. China

E-mail: wangyuhai1516@163.com

${ }^{*}$ Contributed equally

Key words: dexmedetomidine, traumatic brain injury, early brain injury, autophagy, neuroinflammation
TBI. The neuroprotective capacity of DEX is partly dependent on the ROS/nuclear factor erythroid 2-related factor 2 signaling pathway. Taken together, the results of the present study indicated that DEX improves neurological outcomes in mice and reduces neuronal death by protecting against neural autophagy and neuroinflammation.

\section{Introduction}

Traumatic brain injury (TBI) is a major public health problem and a major cause of mortality and disability that imposes a substantial economic burden worldwide, A previous study reported that there were $\sim 288,000$ TBI-related hospitalizations and >56,000 TBI-related deaths in the US alone, and the global economic burden of TBI was estimated at $\sim \$ 400$ billion per annum $(1,2)$. TBI has a high incidence in low-income and middle-income countries, as well as developing countries such as Iran and China (3-5). The incidence of TBI is increasing rapidly due to the significant increase in road traffic collisions, including motor vehicle accidents (5). Although an increasing number of randomized controlled trials including intracranial pressure monitoring, therapeutic hypothermia, surgical methods and drug administration have been performed in recent years and the long-term outcome has substantially improved, a significant benefit is not observed following drug interventions (4-10). Hence, studies aiming to further clarify the pathophysiological mechanisms of TBI and search for new and effective pharmacological intervention targets are important and necessary. The pathophysiology of TBI includes several different physiological changes and mainly involves primary brain injury and secondary brain injury, which lead to neuronal death, neurological deficits and mortality following TBI (11). Primary brain injuries lead to brain tissue disorganization, intracerebral hemorrhage and blood-brain barrier (BBB) damage, which is a direct physical injury to brain tissue that is difficult to prevent and usually cannot be reversed. Secondary brain injury, including calcium overload, oxidative stress, neuroinflammation, autophagy, lipid peroxidation and apoptosis, can be reversed $(12,13)$.

Autophagy is the main cellular lysosomal degradation mechanism for degrading and recycling intracellular proteins and organelles under different physiological and pathological 
conditions (14). Autophagy has been reported to have a core role in many central nervous system diseases, including acute brain injury $(12,15,16)$, intracerebral hemorrhage (17), subarachnoid hemorrhage (SAH) (18) and Huntington's disease (19). Tang et al (15) reported significant decreases in neural apoptosis and necrotic cell death following autophagy was inhibited by fibroblast growth factor- 2 and the autophagy activator rapamycin aggravated brain injury. According to Fang et al (16), activation of autophagy decreases mitochondrial apoptosis, improves neurological function, decreases cerebral edema and alleviates the disruption of the BBB following TBI in mice. Currently, the neuroprotective effect of the activation or inhibition of autophagy remains to be elucidated. A further study of new potential drug targets in the autophagy pathway is required.

Dexmedetomidine (DEX) is a highly selective $\alpha$-2-adrenergic receptor agonist that provides sedation and analgesic effects with minimal respiratory depression (20). It is widely used in surgical procedures and to prevent postoperative delirium $(12,21)$. Recent studies have confirmed that DEX exerts its protective effects on various organ injuries $(12,20)$. Based on accumulating evidence, DEX also improves neurological function and delirium in patients (21) and alleviates EBI in the TBI model and the effect of DEX is dose-dependent $(12,22,23)$. Huang and Hao (22) report that DEX relieves early brain injury (EBI) by inhibiting inflammation and decreasing neuronal apoptosis, which may depend on the TLR4/NF- $\kappa \mathrm{B}$ signaling pathway. Li et al (24) also report that DEX alleviates EBI by inhibiting inflammation through the nuclear factor erythroid 2-related factor 2 (Nrf2) signaling pathway. As shown in the study by Zhao et al (25), DEX increases autophagy, decreases reactive oxygen species (ROS) production and apoptosis and then eliminates damaged mitochondria in a lipopolysaccharide-induced acute kidney injury model through the PI3K/AKT/mTOR pathway. The AKT/mTOR signaling pathway is generally acknowledged as the most important regulatory pathway in autophagy $(26,27)$. ROS regulate the NFE2L2 (nuclear factor, erythroid derived 2, like 2) pathway, transcriptionally activates hypoxia-inducible factor (HIF-1) and p53 and then promotes autophagy $(28,29)$. Our previous studies revealed an important role for $\mathrm{Nrf} 2$ in neuronal death in diseases of the central nervous system $(30,31)$. However, researchers have not determined whether DEX attenuates EBI by regulating autophagy via the ROS/Nrf2 signaling pathway.

The present study constructed a mouse TBI model to study the effects of DEX on EBI and explored the crosstalk between autophagy and inflammation. It also explored the mechanism by which the ROS/Nrf2 signaling pathway may regulate this process.

\section{Materials and methods}

Animals. All animal experiments performed in this study complied with the National Institutes of Health guidelines for the handling of laboratory animals (32) and were approved by the Ethics Committee of the Wuxi Medical College of Anhui Medical University (approval no. YXLL-2020-112). All experiments were conducted on healthy adult male C57BL/6 J mice (age, 8-10 weeks; weight, 22-25 g; Anhui Medical University, Hefei, China). The mice were housed in animal care facilities under environmentally controlled temperature $\left(25 \pm 2^{\circ} \mathrm{C}\right)$ and humidity $(55 \pm 5 \%)$ with 12-h light/dark cycles, and had free access to food and water. The 60 mice were divided into four major groups (15 animals/group): Sham, TBI, TBI + DEX and TBI + DEX + Rap.

Animal TBI model. The TBI model was established in strict accordance with the Feeney weight-drop model of focal injury $(33,34)$. Briefly, the mice were anesthetized with an intraperitoneal injection of $1 \%$ sodium pentobarbital (40 mg/kg) and then placed in a brain stereotaxic apparatus. The rectal temperature was maintained at $37 \pm 0.5^{\circ} \mathrm{C}$ during the operation using a heating pad. Then, a burr hole was made in the left hemisphere at the following coordinates: $0.2 \mathrm{~mm}$ posterior, $1 \mathrm{~mm}$ lateral and $2.2 \mathrm{~mm}$ below the horizontal plane of the bregma. The bone flap was removed to expose the dura mater. The dura was placed under a weight-drop device with an impact sensor. A metal (weight $240 \mathrm{~g}$, tip diameter $3 \mathrm{~mm}$ ) was dropped from $1 \mathrm{~cm}$ above the dura onto the dura mater through a catheter. Then, the scalp was closed and the mice were removed from the apparatus. Finally, the hole was covered with medical bone wax. The animals in the Sham group received similar surgical procedures but without weight-drop impact. At $72 \mathrm{~h}$ following TBI, the mice were sacrificed with $100 \mathrm{mg} / \mathrm{kg}$ sodium pentobarbital via i.p. injection. Mortality was confirmed by observing respiration and by using the corneal reflection method.

Drug administration. DEX was purchased from Jiangsu Hengrui Medicine Co., Ltd., dissolved in $0.9 \%$ sterile saline and administered i.p. at a dose of $20 \mu \mathrm{g} / \mathrm{kg} 2 \mathrm{~h}$ after recovery (12) (Fig. 1A). A dose of $2 \mathrm{mg} / \mathrm{kg}$ rapamycin (Selleck Chemicals; dissolved in 2\% DMSO) was administered i.p. 30 min before TBI induction in the TBI + rapamycin + DEX group.

Modified neurological severity score (mNSS). The severity of brain injury was evaluated by determining neurological function $72 \mathrm{~h}$ after TBI using a previously described neurological grading system (15). The neurological scores of the animals in each group were evaluated by an independent observer. The scoring system consisted of motor, sensory, reflex and balance tests. The neurological scores (mNSS scores) ranged from 0 to 18 points and were calculated by adding the scores together; all mice in each group underwent a behavioral assessment and a higher score represented worse neurological function. All mouse behavior scores were recorded by the same independent observer who was blinded to the study groups.

Brain water content measurement. The severity of brain edema was evaluated by measuring the brain water content using the standard wet-dry method, as previously reported $(18,31,35)$. The mice were sacrificed $72 \mathrm{~h}$ after TBI and the entire brain was harvested and separated into the ipsilateral and contralateral cortices, ipsilateral and contralateral basal ganglia and cerebellum (wet weight). Then, brain specimens from each group were dehydrated at $105^{\circ} \mathrm{C}$ for $24 \mathrm{~h}$ to acquire the dry weight. The percentage of brain water content was equal to (wet weight-dry weight)/wet weight $\times 100 \%$.

Evans blue extravasation. Evans blue extravasation was performed as previously described (36). Briefly, mice were 
anesthetized by $1 \%$ sodium pentobarbital $(50 \mathrm{mg} / \mathrm{kg})$ injection $72 \mathrm{~h}$ after TBI. Evans blue dye (2\%, $5 \mathrm{ml} / \mathrm{kg}$; Sigma-Aldrich; Merck KGaA) was injected into the left femoral vein over $2 \mathrm{~min}$ and circulated for $60 \mathrm{~min}$. Then, the mice were sacrificed with $100 \mathrm{mg} / \mathrm{kg}$ sodium pentobarbital via i.p. injection and with phosphate-buffered saline (PBS) intracardial perfusion. Mortality was identified by observing respiration and by using the corneal reflection method. The brains were removed and quickly divided into the left and right cerebral hemispheres, weighed, homogenized in saline and centrifuged at $15,000 \mathrm{x} \mathrm{g}$ for $30 \mathrm{~min}$ at room temperature. Subsequently, the resultant supernatant was added with an equal volume of trichloroacetic acid, incubated overnight at $4^{\circ} \mathrm{C}$ and centrifuged at $15,000 \mathrm{x} \mathrm{g}$ for $30 \mathrm{~min}$ at room temperature. Next, the resultant supernatant was collected and spectrophotometrically quantified at $610 \mathrm{~nm}$ for Evans blue dye.

Cytokine measurements. IL-1 $\beta$ (cat. no. ab197742; Abcam), IL-6 (cat. no. ab222503; Abcam), TNF- $\alpha$ (cat. no. ab208348; Abcam) and NF-кB (cat. no. ab176663; Abcam) levels were measured using ELISAs according to the manufacturer's instructions.

Analysis of ROS. The nonfluorescent diacetylated 2',7'-dichlorofluorescein (DCF-DA) probe (Sigma-Aldrich; Merck $\mathrm{KGaA}$ ), which becomes highly fluorescent upon oxidation, was used to evaluate intracellular ROS production according to the manufacturer's instructions.

Analysis of lipid peroxidation. Malondialdehyde (37) levels were detected with a lipid peroxidation (37) assay kit (Ex/Em 532/553 nm, cat. no. ab118970; Abcam) according to the manufacturer's instructions.

TdT-mediated dUTP-biotin nick end labeling (TUNEL) assay. A TUNEL assay was conducted to assess neuronal death in the hippocampus. The TUNEL reaction mixture $(50 \mu \mathrm{l})$ was added to each sample and the slides were incubated in a humidified chamber for $60 \mathrm{~min}$ at $37^{\circ} \mathrm{C}$ in the dark. The slides were then incubated with DAPI $(0.1 \mathrm{mg} / \mathrm{ml})$ for $5 \mathrm{~min}$ at room temperature in the dark to stain the nuclei, followed by imaging with a fluorescence microscope. The procedure was performed with a TUNEL staining kit according to the manufacturer's instructions (cat. no. TUN11684817, Roche Diagnostics GmbH). A negative control (without the TUNEL reaction mixture) was used. The apoptotic index (\%) was calculated as the ratio of the number of TUNEL-positive cells/total number of cells $\mathrm{x} 100$. The cell count was confirmed in four randomly selected high-power fields and the data obtained from each field were averaged (magnification, $\mathrm{x} 400$ ).

Western blot analysis. Western blot analyses were performed as previously described (18). Briefly, cerebral cortex samples were collected, homogenized and total protein was extracted using RIPA buffer (CoWin Biosciences). A BCA Protein Assay kit (Beyotime Institute of Biotechnology) was used to measure protein concentrations with the bicinchoninic acid method. Total protein $(30 \mu \mathrm{g})$ was separated via $12 \%$ SDS-PAGE and transferred onto PVDF membranes. The membranes were blocked with $5 \%$ nonfat milk at room temperature for $1 \mathrm{~h}$. The
A
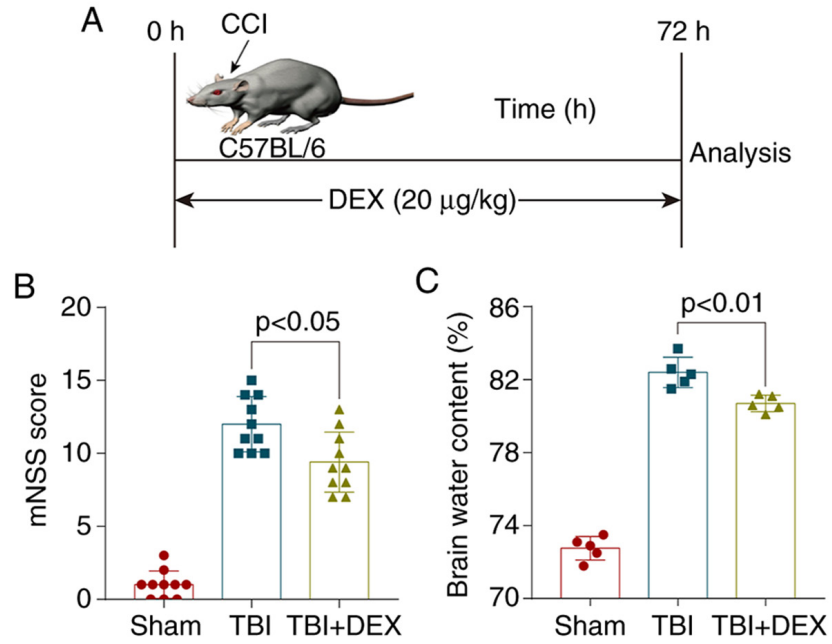

Figure 1. DEX alleviates neurological deficits and brain edema following TBI. (A) Schematic of the experimental paradigm for establishing the TBI model in mice. (B) Neurological scores of mice in the sham group, TBI group and TBI group treated with DEX at $72 \mathrm{~h}$ after TBI $(\mathrm{n}=10, \mathrm{P}<0.05)$. (C) Comparison of the brain water content between the three groups $(n=5$; $\mathrm{P}<0.01)$. DEX, dexmedetomidine; TBI, traumatic brain injury; mNSS, modified neurological severity score.

membranes were then incubated with the following primary antibodies overnight at $4^{\circ} \mathrm{C}$ : Rabbit anti- $\beta$ actin $(1: 1,000$; rabbit polyclonal; cat. no. ab8227), rabbit anti-caspase-3 (1:2,000; rabbit polyclonal; cat. no. ab184787), rabbit anti-Nrf2 (1:1,000; rabbit polyclonal; cat. no. ab31163), rabbit anti-heme oxygenase (HO)-1 (1:1,000; rabbit polyclonal; cat. no. ab13243), rabbit anti-Beclin-1 (1 $\mu \mathrm{g} / \mathrm{ml}$, rabbit monoclonal; cat. no. ab62557) and rabbit anti-LC-3B (1 $\mu \mathrm{g} / \mathrm{ml}$, rabbit monoclonal; cat. no. ab48394; all from Abcam). After washing the membranes with TBST (0.5\% Tween-20) three times at room temperature for $20 \mathrm{~min}$, HRP-conjugated goat anti-rabbit IgG or goat anti-mouse IgG secondary antibodies (1:2,000; cat. no. 7074s; Cell Signaling Technology, Inc.) were applied and the membranes were incubated with the secondary antibodies at room temperature for $1.5 \mathrm{~h}$. The protein bands were detected using a Bio-Rad imaging system (Bio-Rad Laboratories, Inc.) and quantified with ImageJ software (version 1.52; National Institutes of Health).

Statistical analysis. The data are reported as the means \pm standard error of mean. SPSS v14.0 (SPSS, Inc.) and GraphPad Prism 6 (GraphPad Software, Inc.) software were used for the statistical analyses. Student's t-test (unpaired) was used if two groups were compared and one-way analysis of variance (ANOVA) followed by Bonferroni's post hoc test was used if two independent variables were compared. For nonnormally distributed data and/or data with a nonhomogeneous variance, the Kruskal-Wallis test followed by Dunn's post hoc test was used. $\mathrm{P}<0.05$ was considered to indicate a statistically significant difference.

\section{Results}

DEX alleviates neurological deficits and brain edema following TBI. The modified neurological severity score (mNSS) was calculated to evaluate neurological deficits and the brain water content determined using the wet-dry method at $72 \mathrm{~h}$ after 
A
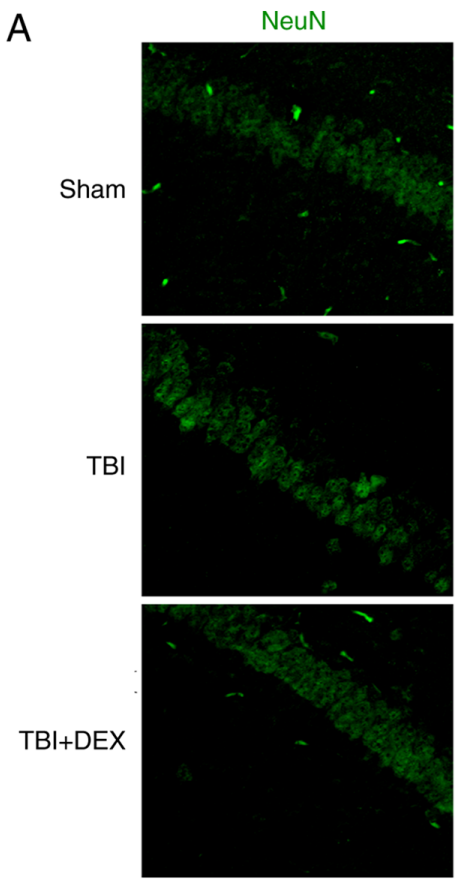

B
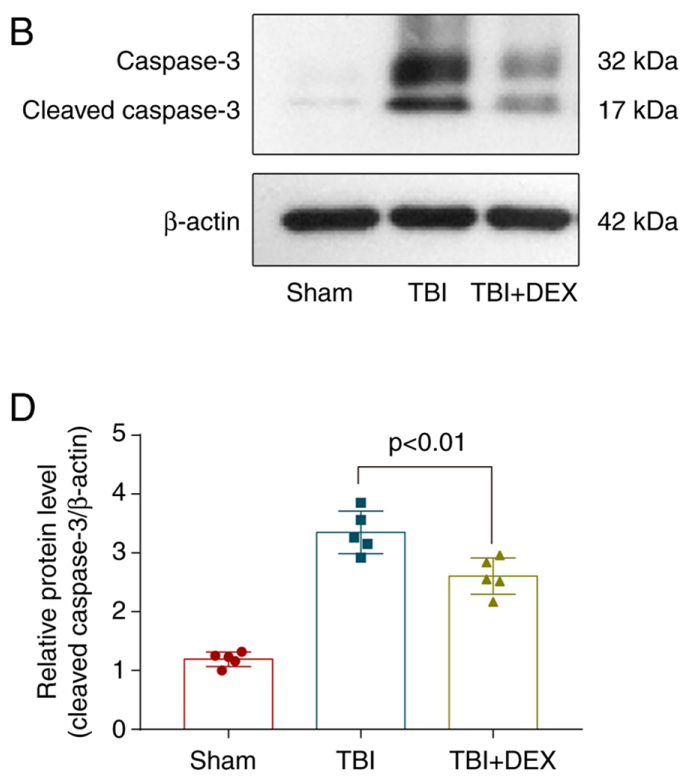

DAPI
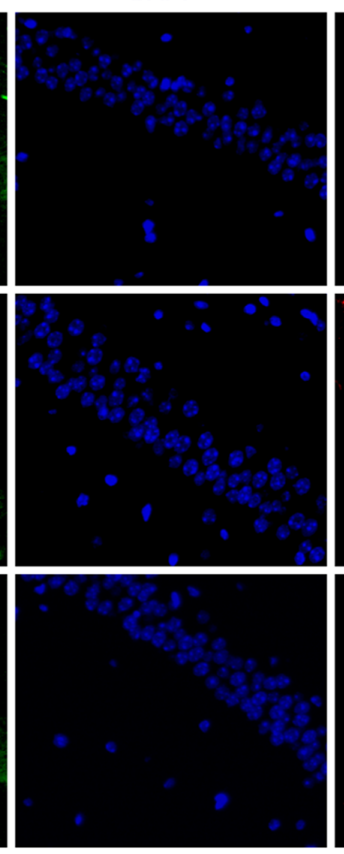

C

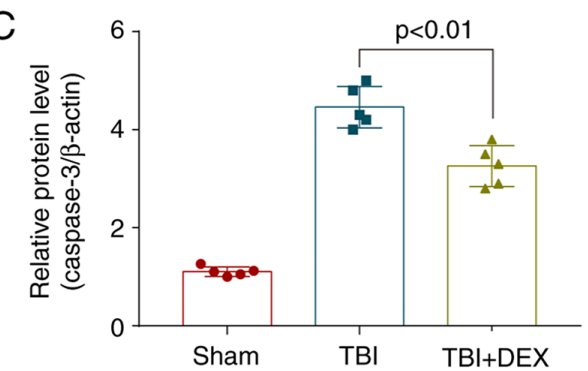

E

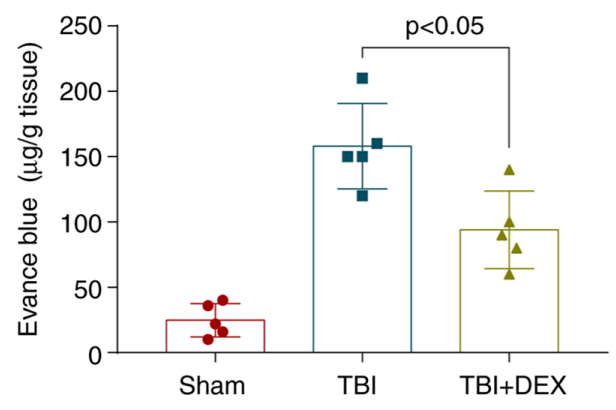

Figure 2. DEX alleviates neuronal apoptosis and increased BBB permeability following TBI. (A) TUNEL staining showed that DEX alleviated neuronal apoptosis in the hippocampus at $72 \mathrm{~h}$ after TBI and representative images of apoptotic neurons are shown. Scale bar $=50 \mu \mathrm{m}$. (B) Levels of caspase-3 and cleaved caspase-3 in the brain cortex of mice following TBI were determined using western blotting. Quantification of (C) caspase-3 and (D) cleaved caspase-3 in the brain cortex relative to $\beta$-actin, the loading control. DEX reduced caspase- 3 and cleaved caspase- 3 levels in mice with TBI. (E) DEX reduces BBB permeability $(\mathrm{n}=5, \mathrm{P}<0.05$; ANOVA; means \pm standard error of mean). DEX, dexmedetomidine; BBB, blood-brain barrier; TBI, traumatic brain injury; TUNEL, terminal deoxynucleotidyl transferase dUTP nick end labeling; DAPI, 4'6-diamidino-2-phenylindole.

TBI to evaluate brain damage and clarify the neuroprotective effect of DEX on TBI. TBI significantly increased the neurological scores and DEX administration significantly improved neurological function (Fig. 1B). Similar results were obtained for brain water content, which were increased significantly after $\mathrm{TBI}$ and was alleviated by DEX treatment $(\mathrm{P}<0.05$; Fig. 1C).

DEX alleviates neuronal apoptosis and BBB permeability following TBI. Neuronal apoptosis and BBB permeability are the main factors that lead to EBI following TBI. Therefore, a TUNEL assay was used to evaluate the level of cell death in TBI mice treated with and without DEX at $72 \mathrm{~h}$ after model construction. The expression levels of apoptosis-related proteins were detected using Western blotting. Evans blue extravasation was analyzed to clarify BBB permeability. The results revealed more hippocampal neuronal death following TBI and DEX decreased neuronal apoptosis (Fig. 2A). The western blot results also indicated that DEX reduced the expression levels of the apoptosis-related protein caspase-3 (Fig. 2B-D). Compared with the sham and TBI groups, DEX 

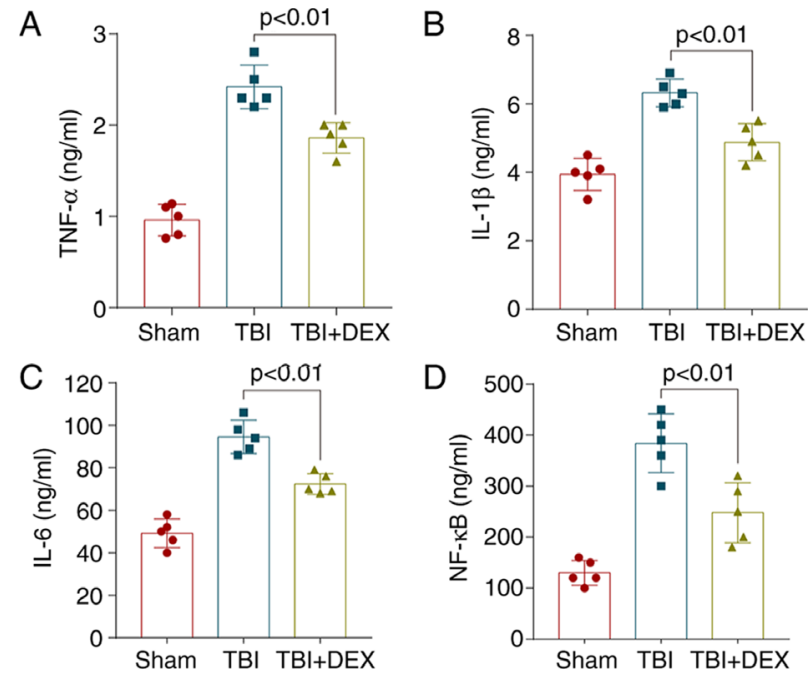

Figure 3. DEX alleviates neuroinflammation following TBI. DEX significantly reduced hippocampal (A) TNF- $\alpha$, (B) IL-1 $\beta$, (C) IL-6 and (D) NF- $\kappa B$ levels at $72 \mathrm{~h}$ after TBI $(\mathrm{n}=5, \mathrm{P}<0.05$, ANOVA; means \pm standard error of mean). DEX, dexmedetomidine; TBI, traumatic brain injury.

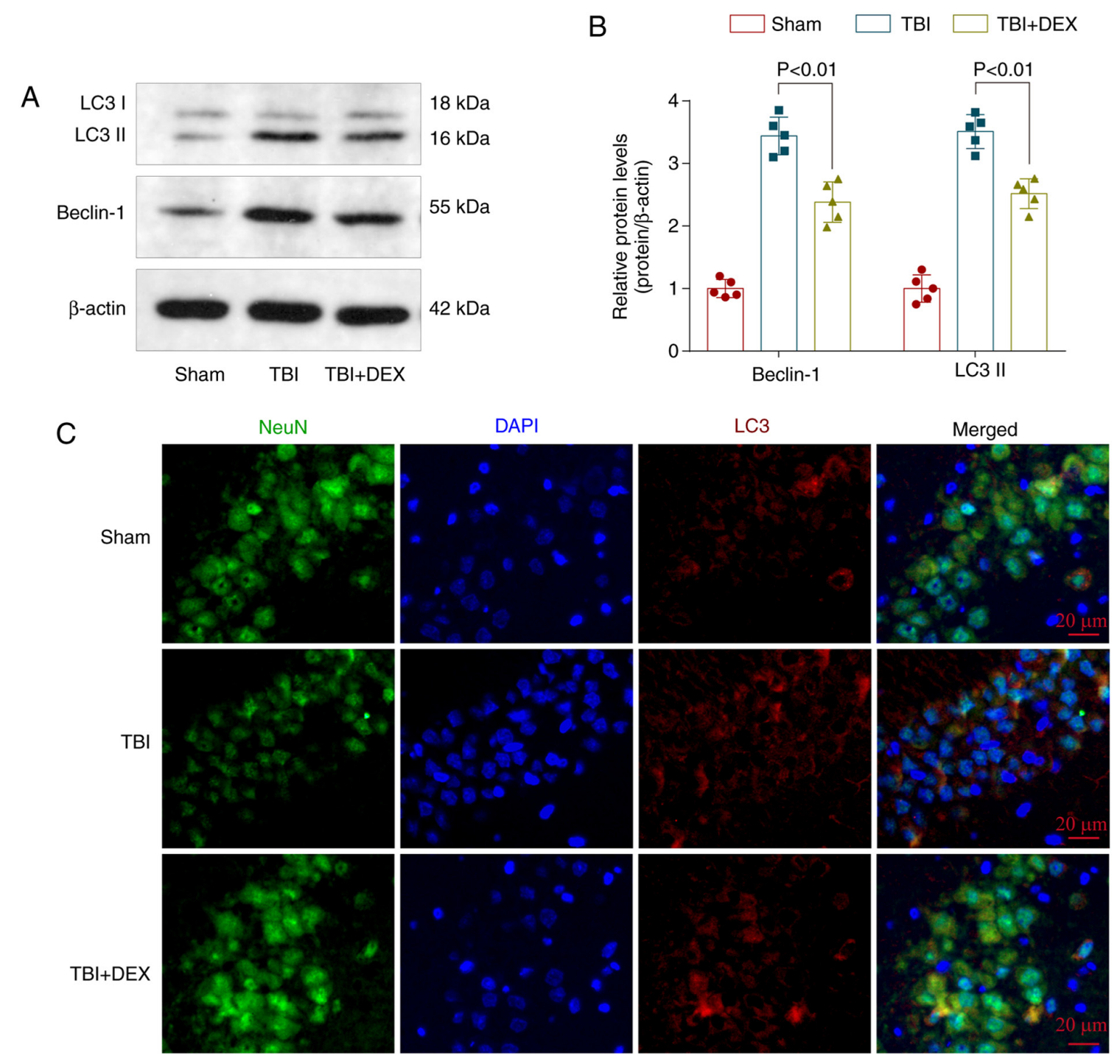

Figure 4. DEX inhibits TBI-induced autophagy activation in the hippocampus. (A) The expression of LC3 and Beclin-1 in the brain cortex of mice with TBI was determined using western blotting. (B) Quantification of LC3 and Beclin-1 levels in the brain cortex relative to $\beta$-actin, the loading control. DEX reduced LC3 and Beclin-1 expression in mice with TBI ( $\mathrm{n}=5, \mathrm{P}<0.01$; ANOVA; means \pm standard error of mean). (C) Representative pictures of double staining for LC3 and NeuN in the injured hippocampus following TBI. LC3-positive neurons were rarely observed in the hippocampus of the sham group and DEX decreased the number of LC3-positive neurons. Scale bar=20 $\mu \mathrm{m}$ (magnification, x400). DEX, dexmedetomidine; TBI, traumatic brain injury. 

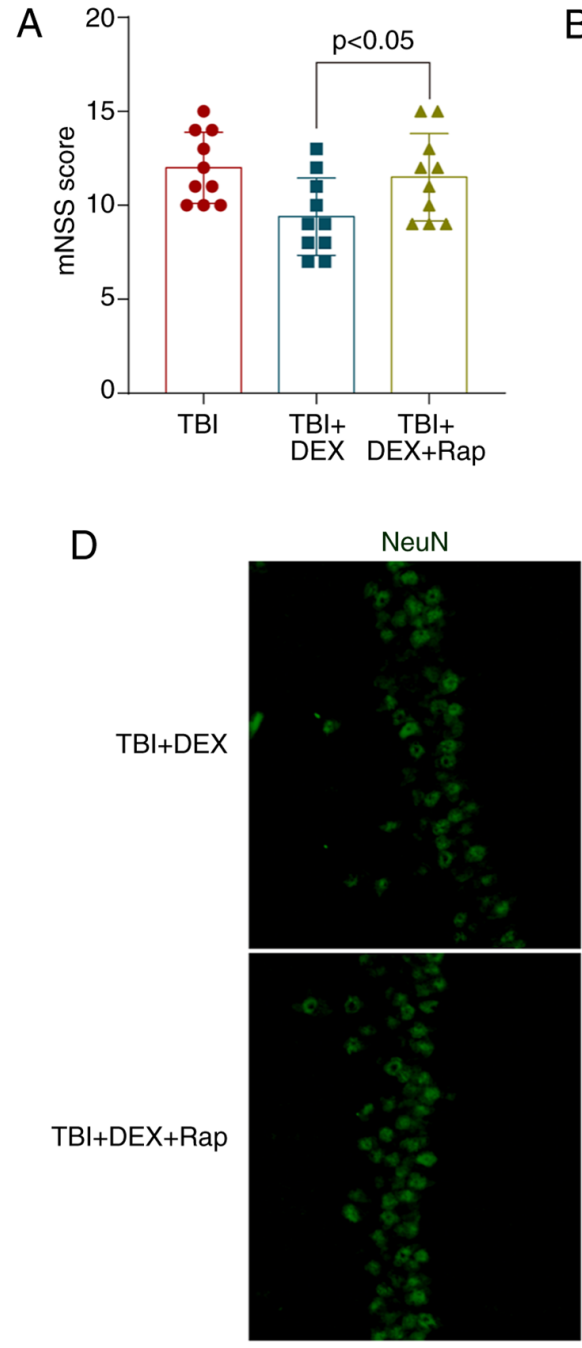

$\mathrm{E}$

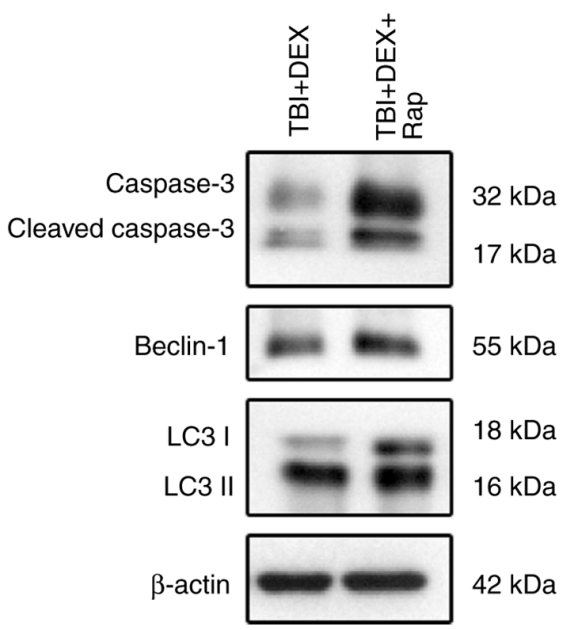

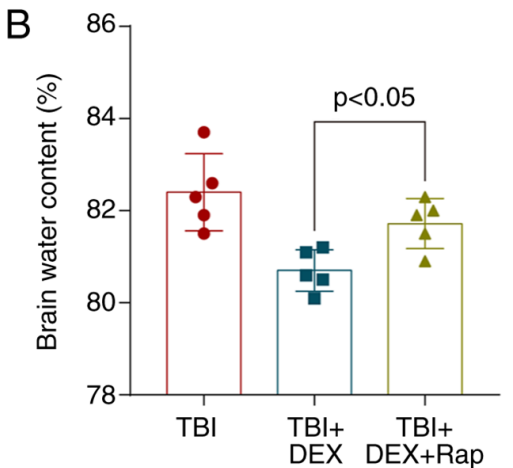
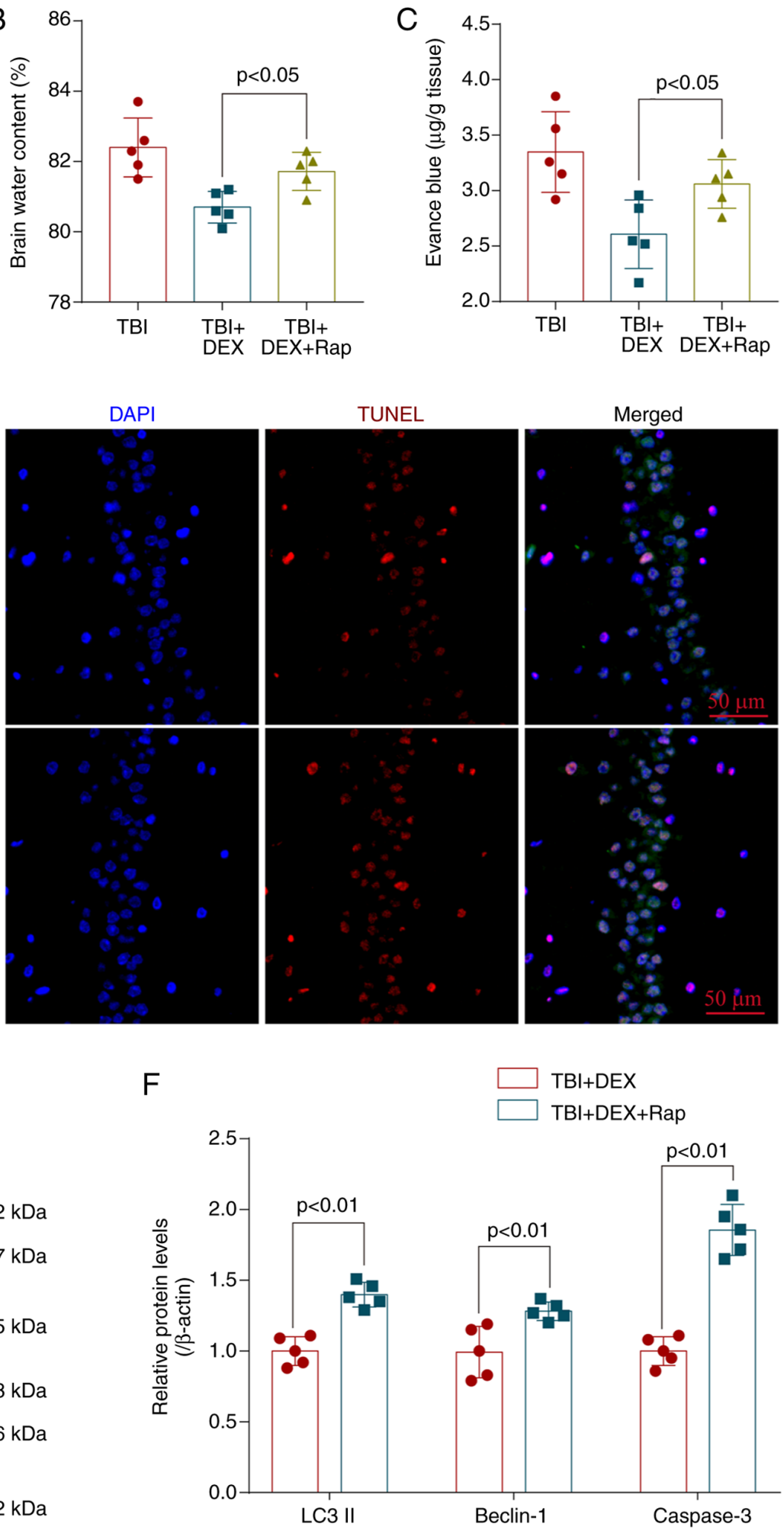

Figure 5. Rap stimulates autophagy and reverses the neuroprotective effect of DEX. Pretreatment with rapamycin significantly increased the (A) mNSS, (B) The brain water content and (C) BBB permeability compared with the TBI + DEX group $(n=10, P<0.05)$. (D) Rapamycin increased neuronal apoptosis in the injured hippocampus compared with the TBI + DEX group (magnification, x400). (E) Levels of LC3, Beclin-1 and caspase-3 in the brain cortex of mice with TBI were determined using western blotting. (F) Quantification of LC3, Beclin-1 and caspase-3 levels in the brain cortex relative to $\beta$-actin, the loading control. Rap increases the LC3, Beclin-1 and caspase-3 levels in DEX-treated mice following TBI ( $n=5$, ANOVA; means \pm standard error of mean). Rap, rapamycin; DEX, dexmedetomidine; mNSS, modified neurological severity score; TBI, traumatic brain injury; BBB, blood-brain barrier; DAPI, 4'6-diamidino-2-phenylindole.

significantly decreased the extent of damage to the blood-brain barrier (Fig. 2E). Based on these results, DEX exerted neuroprotective effects following TBI.
DEX alleviates neuroinflammation following TBI. As previous studies have identified a vital role for neuroinflammation in EBI following TBI, increased neuroinflammation 
A

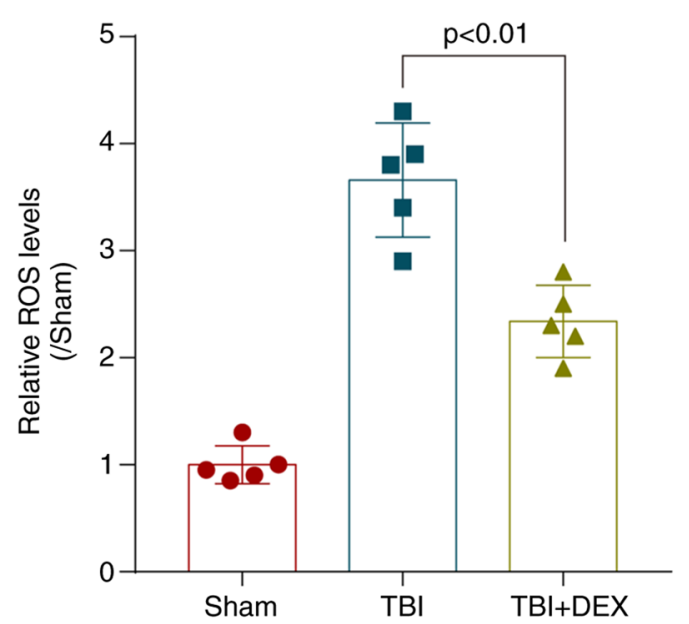

B

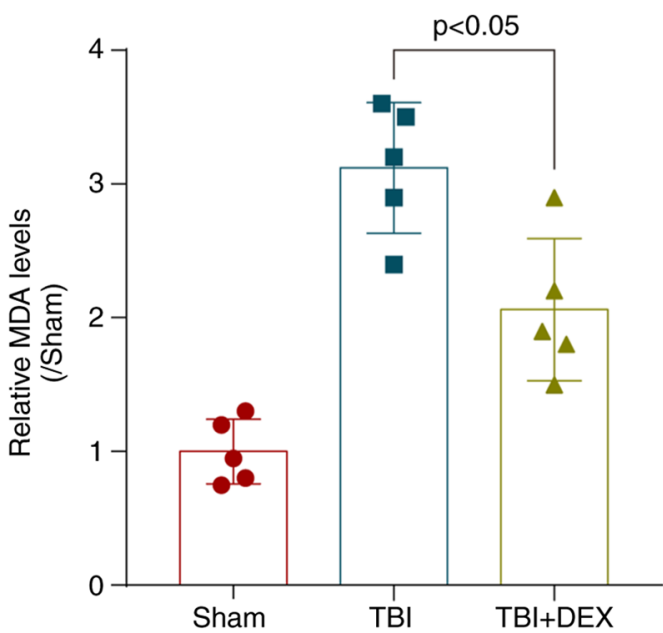

C

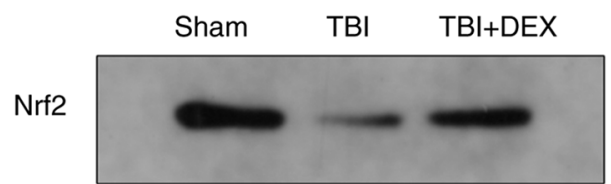

$68 \mathrm{kDa}$

$\mathrm{HO}-1$

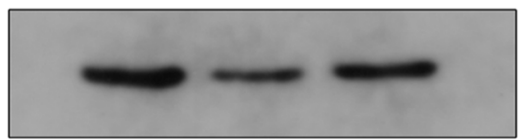

$33 \mathrm{kDa}$

$\beta$-actin

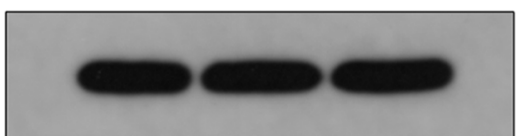

$42 \mathrm{kDa}$
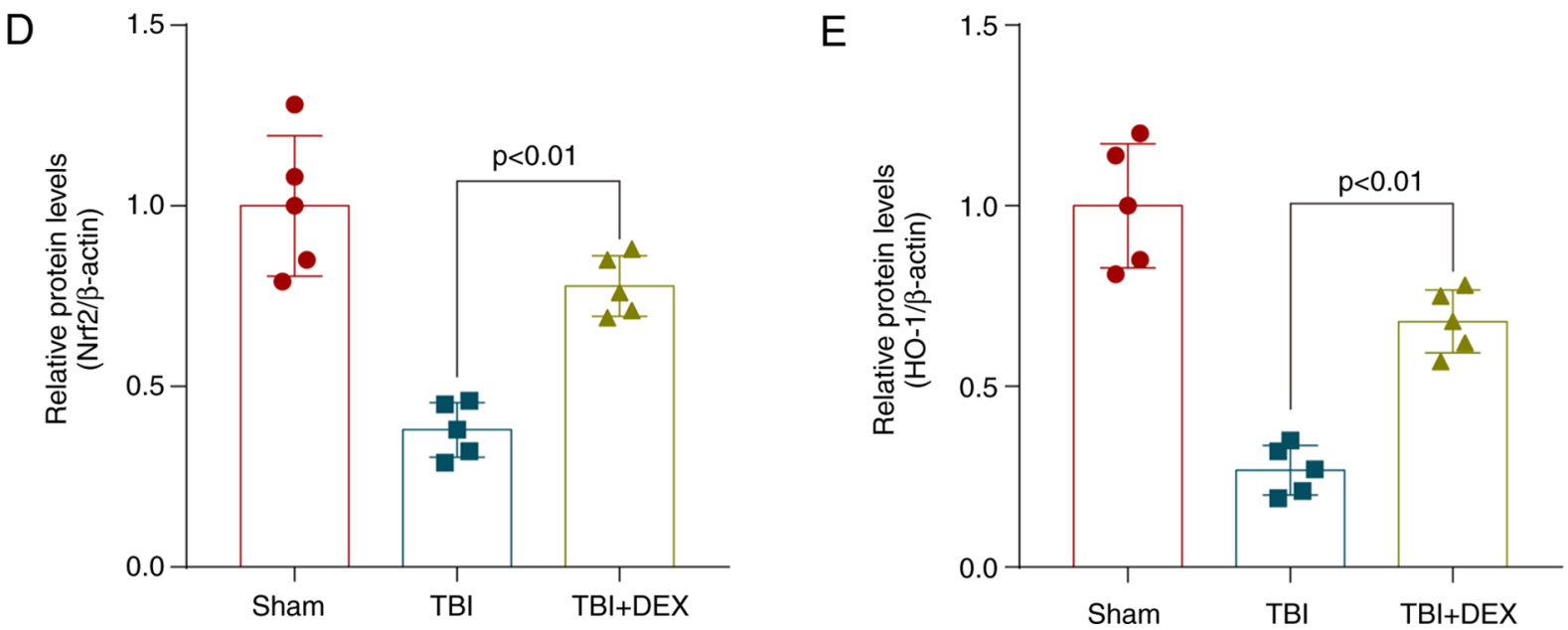

Figure 6. DEX regulates autophagy following TBI by modulating the ROS/Nrf2 signaling pathway. A-B: The levels of (A) ROS and (B) MDA were increased following TBI and decreased significantly following DEX treatment. (C) The expression of Nrf2 and HO-1 in the brain cortex of mice with TBI was determined using western blotting. Quantification of (D) Nrf2 and (E) HO-1 levels in the brain cortex relative to the $\beta$-actin loading control. DEX increased Nrf2 and HO-1 expression in mice with TBI $(n=5, \mathrm{P}<0.01$; ANOVA; mean \pm SEM). DEX, dexmedetomidine; TBI, traumatic brain injury; ROS, reactive oxygen species; Nrf2, nuclear factor erythroid 2-related factor 2; MDA, malondialdehyde; HO, heme oxygenase.

aggravates EBI $(12,20,22,24)$. The inflammatory complex induces the secretion of pro-inflammatory cytokines, including IL-1 $\beta$, IL- 6 and TNF- $\alpha$ and the subsequent activation of pro-inflammatory signaling through $\mathrm{NF}-\kappa \mathrm{B}$ to initiate pyroptosis. Therefore, the hippocampal levels of IL- $1 \beta$, IL- 6 , TNF- $\alpha$ and NF- $\kappa$ B were measured using ELISAs. The levels of the pro-inflammatory cytokines were increased significantly following TBI, while their levels decreased significantly following DEX treatment (Fig. 3A-D). Hence, these results suggested that DEX exhibited potent anti-inflammatory activity against TBI-induced neuroinflammation.

DEX inhibits TBI-induced autophagy activation in the hippocampus. To clarify whether autophagy serves an important role in TBI and the regulatory effect of DEX 


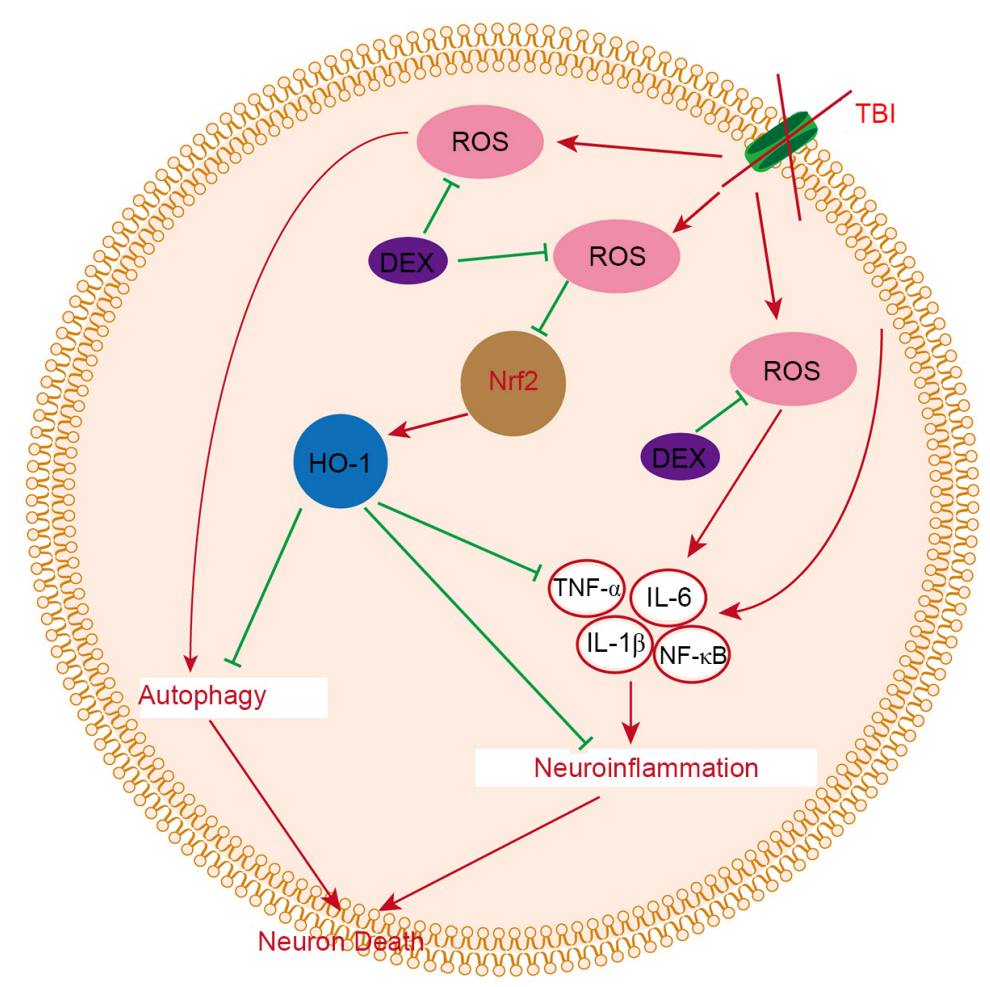

Figure 7. A diagram of the proposed model explaining the observations of ROS/Nrf2-mediated regulation of autophagy following TBI and potential mechanisms underlying the effect of the DEX intervention. ROS, reactive oxygen species; Nrf2, nuclear factor erythroid 2-related factor 2; TBI, traumatic brain injury; HO, heme oxygenase; DEX, dexmedetomidine.

on autophagy, the levels of the autophagy-related proteins Beclin-1 and LC3 in each group were detected using western blotting (Fig. 4A). The levels of both Beclin-1 and LC3 were increased following TBI but decreased significantly following DEX treatment (Fig. 4B). LC3 is a biomarker for autophagy activation and LC3 was chosen to mark autophagic cells. Immunofluorescence staining showed few LC3-positive neurons in the hippocampus of the Sham group, but were widespread in the hippocampus following TBI induction. However, the number of LC3-positive neurons decreased following DEX administration (Fig. 4C).

Rapamycin stimulates autophagy and reverses the neuroprotective effect of DEX. Rapamycin is a specific activator of autophagy (18). Mice were pretreated with rapamycin before the induction of TBI to investigate the relationship between autophagy and the neuroprotective effect of DEX. Pretreatment with rapamycin dramatically enhanced neurological deficits (Fig 5A), aggravated brain edema (Fig 5B) and BBB permeability (Fig 5C) and reversed the neuroprotective effect of DEX. Additionally, the TUNEL assay also showed that rapamycin also significantly increased neuronal apoptosis in the injured hippocampus compared with the TBI + DEX group (Fig 5D). Levels of autophagy-related proteins and apoptosis-related proteins were detected using western blotting (Fig. 5E). DEX significantly decreased the levels of caspase 3, Beclin-1 and LC3 (Fig. 5F) but these changes were partially blocked by rapamycin administration. Thus, rapamycin activated autophagy, abolished the anti-autophagy effects of DEX and reversed the neuroprotective effects of DEX on TBI.
DEX regulates autophagy by modulating the ROS/Nrf2 signaling pathway following TBI. AKT/mTOR is a core signaling pathway of cell autophagy. According to previous studies, activation of the AKT/mTOR signaling pathway is partially dependent on ROS production $(38,39)$. The present study explored whether autophagy inhibition by ROS occurred through the ROS/Nrf2 signaling pathway following DEX treatment. ROS levels were detected with a DCF-DA probe and the degree of membrane lipid peroxidation evaluated by measuring the MDA content. The levels of ROS and MDA were significantly increased following TBI but decreased following DEX treatment (Fig. 6A and B). The levels of the Nrf2 and HO-1 protein were detected by performing western blotting to investigate neuronal autophagy (Fig. 6C). The levels of Nrf2 and HO-1 were decreased significantly in the TBI group and increased following DEX administration (Fig. 6D and E). Thus, these results showed that DEX may inhibit TBI-induced autophagy by regulating the ROS/Nrf2 signaling pathway.

\section{Discussion}

The present study evaluated the therapeutic potential of DEX to alleviate EBI in a mouse model of TBI. It was demonstrated that DEX is a neuroprotective agent that attenuated EBI following TBI. It was found that DEX i) improved neurological dysfunction following TBI, ii) alleviated brain damage in a mouse TBI model, iii) relieved neuroinflammation following TBI and then decreased inflammatory damage in the brain and iv) prevented autophagy following TBI and alleviated neuronal death, and v) the anti-neuroinflammatory and anti-autophagy effects of DEX may be related to the ROS/Nrf2 pathway (Fig. 7). 
The present study, to avoid bias of biological sex and gonadal hormone status, used healthy adult male C57BL/6J mice not females. The main reason was that studies indicate that biological sex and sex hormone levels may affect the outcome following TBI $(40,41)$. A recent study also recommends that studies should include information on gonadal hormone status and need to include biological sex and gonadal hormone status in the design and analysis following TBI (42).

DEX, a highly selective $\alpha$-2-adrenergic receptor agonist with anxiolytic, sedative and analgesic properties, has been widely used as an adjuvant during general anesthesia and anti-delirium with minimal respiratory depression $(12,21)$. Clinical studies have shown that the administration of low-dose DEX to critically ill adults reduces the incidence of delirium during stays in intensive care units and reduces cognitive decline for up to one postoperative month in elderly patients undergoing scheduled laparotomy $(21,43,44)$, but the specific neuroprotective mechanism remains to be elucidated Chen et al (45) aimed to further clarify the mechanisms and report that DEX protects against MTX-induced cognitive impairment by inhibiting neuronal toxicity and inflammation. In hypoxia-activated BV2 microglia and neonatal rats subjected to hypoxia, DEX decreases hippocampal synaptic loss and neuronal damage and the potential mechanisms may be that DEX prevents hypoxia-induced microglial NOX2 activation and then inhibits oxidative stress and the neuroinflammatory response (46). Mei et al (47) also report that DEX alleviates BBB disruption, learning and memory impairments, systemic inflammation and neuroinflammation in a cecal ligation and puncture (CLP)-induced septic model. Hence, the anti-inflammation and anti-neuroinflammation effects serve an important role in neuroprotection following DEX treatment. As shown in the present study, DEX alleviated neuroinflammation in mouse TBI models. Additionally, DEX directly regulates several cell death models $(22,25,48-51)$. According to Gao et al (49), DEX induces the expression of the neuroglobin protein in hippocampal tissues and then inhibits neuronal apoptosis through the mitochondrial pathway. Sun et al (50) indicate that DEX may preserve brain function and ultimately improve the outcome of sepsis by decreasing pyroptosis and subsequently protecting neurons. In Shen et al (51), DEX postconditioning also improves cardiac outcomes and neurological function following cardiac arrest and resuscitation in swine, partly by suppressing cell necroptosis. Zhao et al (25) report that DEX alleviates acute kidney injury by increasing autophagy through the modulation of the PI3K/AKT/mTOR pathway. At present, the neuroprotective effect of DEX-regulated autophagy activation or inhibition remains to be elucidated.

Autophagy regulates the turnover of cellular constituents to ensure the removal and recycling of toxins and is important in cell homeostasis (52). The role of autophagy has been confirmed in many central nervous system diseases, including acute brain injury $(12,15,16)$, intracerebral hemorrhage (17), SAH (18) and Huntington's disease (19). Autophagy transports materials in cells to lysosomes for degradation through different pathways involved in the regulation of cell survival and death mechanisms following TBI (53). Therefore, autophagy serves a very important role in neuronal injury and repair following TBI. Xue et al (54) report that DEX improves long-term learning cognitive function by inhibiting neuronal autophagy in a neonatal rat model of hypoxic-ischemic brain injury. As shown in Li et al (12), DEX reverses upregulated circLrplb and Dram 2 expression and downregulates miR-27a-3p expression to subsequently inhibit excessive autophagy and improve the TBI-induced neurological impairment. Based on the findings reported by Zhao et al (25), DEX promotes cell autophagy and subsequent LPS-induced acute kidney injury. Yang et al (20) also report that the protective effect of DEX is based on the activation of autophagy through the $\alpha 2$-adrenoreceptor/AMPK/mTOR pathway. In the present study, autophagy was excessively activated following TBI and then led to neurological impairments, BBB disruption, brain edema and neuronal apoptosis, which were reversed by the DEX treatment.

The mechanisms and molecules regulating the autophagy pathway are complex and involve mTOR-dependent and nondependent pathways. The upstream regulatory pathway of mTOR includes AMPK and the PI3K/AKT pathway $(13,20)$. As shown in the present study, DEX decreased levels of ROS production, subsequently inhibiting the activation of autophagy. In the nucleus, ROS regulate the Nrf2 pathway, transcriptionally activate hypoxia-inducible factor (HIF-1) and p53 and then promote autophagy $(28,29)$. Wang et al $(55)$ report that rehmapicrogenin improves adriamycin-induced nephropathy in vivo and in vitro and the mechanisms may be to reduce ROS accumulation and then alter the expression levels of Nrf2. Nrf2 regulates the expression of $>250$ genes through a specific binding site. The majority of these genes regulate oxidative stress and cell apoptosis, autophagy and ferroptosis (30). TBI leads to intracellular ROS accumulation and decreases the expression levels of Nrf2 and $\mathrm{HO}-1$ and the Nrf2/HO-1 signaling pathway also directly regulates autophagy (56). In the present study, DEX decreased ROS levels and then downregulated Nrf2 and HO-1 expression in the experimental TBI model. DEX ameliorated EBI following TBI by inhibiting neuroinflammation and autophagy via the Nrf2/HO-1 pathway. The specific mechanism remains unclear and other potential molecular mechanisms may serve important roles. Therefore, further research is needed to explore these mechanisms. In addition, the experiments was performed in mice and debate persists regarding whether the treatment is effective in humans. In the future, the clinical effect of DEX will be explored on patients with TBI.

In summary, the present study provided evidence that autophagy, which is mediated by ROS and Nrf2, was an important cellular regulatory mechanism and contributed to EBI following TBI. The present study reported the DEX-mediated regulation of autophagy by the ROS/Nrf2 pathway and provided a new way to explore the biological effects and mechanisms underlying the anti-autophagy, anti-inflammatory and neuroprotective properties of DEX.

\section{Acknowledgements}

Not applicable.

\section{Funding}

The present study was supported. by the National Natural Science Foundation of China (grant. no. 81871589) and the Wuxi Municipal Bureau on Science and Technology (grant no. N20201008). 


\section{Availability of data and materials}

The datasets used and/or analyzed during the current study are available from the corresponding author on reasonable request.

\section{Authors' contributions}

$\mathrm{XF}$ and $\mathrm{WM}$ performed the experiments and wrote the manuscript. XF, JZ, WJ and YW assisted in performing the experiments and prepared all the figures. YW and WM designed the study and revised the manuscript. XF, YW and WM confirm the authenticity of all the raw data All authors read and approved the final manuscript.

\section{Ethics approval and consent to participate}

All animal experiments performed in this study complied with the National Institutes of Health guidelines for the handling of laboratory animals and were approved by the Ethics Committee of the Wuxi Medical College of Anhui Medical University (approval no. YXLL-2020-112).

\section{Patient consent for publication}

Not applicable.

\section{Competing interests}

The authors declare that they have no competing interests.

\section{References}

1. Rowell SE, Meier EN, McKnight B, Kannas D, May S, Sheehan K, Bulger EM, Idris AH, Christenson J, Morrison LJ, et al: Effect of out-of-Hospital tranexamic acid vs placebo on 6-month functional neurologic outcomes in patients with moderate or severe traumatic brain Injury. JAMA 324: 961-974, 2020.

2. Shankar JJ and Vandorpe R: CT perfusion for confirmation of brain death. AJNR Am J Neuroradiol 34: 1175-1179, 2013.

3. Jiang JY, Gao GY, Feng JF, Mao Q, Chen LG, Yang XF, Liu JF, Wang YH, Qiu BH and Huang XJ: Traumatic brain injury in China. Lancet Neurol 18: 286-295, 2019.

4. Chen J, Li M, Chen L, Chen W, Zhang C, Feng Y, Wang Y and Chen Q: The effect of controlled decompression for severe traumatic brain injury: A randomized, controlled trial. Front Neurol 11: 107, 2020.

5. Chen JH, Li PP, Yang LK, Chen L, Zhu J, Hu X and Wang YH: Value of ventricular intracranial pressure monitoring for traumatic bifrontal contusions. World Neurosurg 113: e690-e701, 2018.

6. Nichol A, French C, Little L, Haddad S, Presneill J, Arabi Y, Bailey M, Cooper DJ, Duranteau J, Huet O, et al: Erythropoietin in traumatic brain injury (EPO-TBI): A double-blind randomised controlled trial. Lancet 386: 2499-2506, 2015.

7. Hutchinson PJ,Kolias AG, Timofeev IS, CorteenEA, Czosnyka M, Timothy J, Anderson I, Bulters DO, Belli A, Eynon CA, et al Trial of decompressive craniectomy for traumatic intracranial hypertension. N Engl J Med 375: 1119-1130, 2016.

8. Cooper DJ, Nichol AD, Bailey M, Bernard S, Cameron PA, Pili-Floury S, Forbes A, Gantner D, Higgins AM, Huet O, et al: Effect of early sustained prophylactic hypothermia on neurologic outcomes among patients with severe traumatic brain injury: The POLAR randomized clinical trial. JAMA 320: 2211-2220, 2018.

9. Wright DW, Yeatts SD, Silbergleit R, Palesch YY, Hertzberg VS, Frankel M, Goldstein FC, Caveney AF, Howlett-Smith H, Bengelink EM, et al: Very early administration of progesterone for acute traumatic brain injury. N Engl J Med 371: 2457-2466, 2014
10. Robertson CS, Hannay HJ, Yamal JM, Gopinath S, Goodman JC, Tilley BC; Epo Severe TBI Trial Investigators, Baldwin A, Rivera Lara L, Saucedo-Crespo H, et al: Effect of erythropoietin and transfusion threshold on neurological recovery after traumatic brain injury: A randomized clinical trial. JAMA 312: 36-47, 2014.

11. Wang Y, Wang L, Hu T, Wang F, Han Z, Yin Z, Ge X, Xie K and Lei P: Hydrogen improves cell viability partly through inhibition of autophagy and activation of PI3K/Akt/GSK3 $\beta$ signal pathway in a microvascular endothelial cell model of traumatic brain injury. Neurol Res 42: 487-496, 2020.

12. Li H, Lu C, Yao W, Xu L, Zhou J and Zheng B: Dexmedetomidine inhibits inflammatory response and autophagy through the circLrp1b/miR-27a-3p/Dram2 pathway in a rat model of traumatic brain injury. Aging (Albany NY) 12: 21687-21705, 2020.

13. Wang Y, Zhao M, Shang L, Zhang Y, Huang C, He Z, Luo M, Wu B, Song P, Wang M and Duan F: Homerla protects against neuronal injury via PI3K/AKT/mTOR signaling pathway. Int J Neurosci 130: 621-630, 2020.

14. Ceccariglia S, Cargnoni A, Silini AR and Parolini O: Autophagy: A potential key contributor to the therapeutic action of mesenchymal stem cells. Autophagy 16: 28-37, 2020.

15. Tang C, Shan Y, Hu Y, Fang Z, Tong Y, Chen M, Wei X, Fu X and $\mathrm{Xu} X$ : FGF2 attenuates neural cell death via suppressing autophagy after rat mild traumatic brain injury. Stem Cells Int 2017: 2923182, 2017.

16. Fang J, Zhu Y, Wang H, Cao B, Fei M, Niu W, Zhou Y, Wang X, $\mathrm{Li}$ X and Zhou M: Baicalin protects mice brain from apoptosis in traumatic brain injury model through activation of autophagy. Front Neurosci 12: 1006, 2018.

17. Zhao M, Gao J, Cui C, Zhang Y, Jiang $X$ and Cui J: Inhibition of PTEN ameliorates secondary hippocampal injury and cognitive deficits after intracerebral hemorrhage: Involvement of AKT/FoxO3a/ATG-mediated autophagy. Oxid Med Cell Longev 2021: 5472605, 2021.

18. Chen JH, Wu T, Xia WY, Shi ZH, Zhang CL, Chen L, Chen QX and Wang YH: An early neuroprotective effect of atorvastatin against subarachnoid hemorrhage. Neural Regen Res 15: 1947-1954, 2020.

19. Aron R, Pellegrini P, Green EW, Maddison DC, Opoku-Nsiah K, Oliveira AO, Wong JS, Daub AC, Giorgini F, Muchowski P and Finkbeiner S: Deubiquitinase Usp12 functions noncatalytically to induce autophagy and confer neuroprotection in models of Huntington's disease. Nat Commun 9: 3191, 2018.

20. Yang T, Feng X, Zhao Y, Zhang H, Cui H, Wei M, Yang H and Fan H: Dexmedetomidine enhances autophagy via $\alpha 2-A R / A M P K / m T O R$ pathway to inhibit the activation of NLRP3 inflammasome and subsequently alleviates lipopolysaccharide-induced acute kidney injury. Front Pharmacol 11: 790, 2020.

21. Subramaniam B, Shankar P, Shaefi S, Mueller A, O'Gara B, Banner-Goodspeed V, Gallagher J, Gasangwa D, Patxot M, Packiasabapathy S, et al: Effect of intravenous acetaminophen vs placebo combined with propofol or dexmedetomidine on postoperative delirium among older patients following cardiac surgery: The DEXACET randomized clinical trial. JAMA 321: 686-696, 2019.

22. Huang GR and Hao FG: Dexmedetomidine inhibits inflammation to alleviate early neuronal injury via TLR4/NF- $\mathrm{B}$ pathway in rats with traumatic brain injury. Crit Rev Eukaryot Gene Expr 31: 41-47, 2021.

23. Bilodeau V, Saavedra-Mitjans M, Frenette AJ, Burry L, Albert M, Bernard F and Williamson DR: Safety of dexmedetomidine for the control of agitation in critically ill traumatic brain injury patients: A descriptive study. J Clin Pharm Ther 46: 1020-1026, 2021.

24. Li F, Wang X, Zhang Z, Zhang X and Gao P: Dexmedetomidine attenuates neuroinflammatory-induced apoptosis after traumatic brain injury via Nrf2 signaling pathway. Ann Clin Transl Neurol 6: 1825-1835, 2019.

25. Zhao Y, Feng X, Li B, Sha J, Wang C, Yang T, Cui H and Fan H: Dexmedetomidine protects against lipopolysaccharide-induced acute kidney injury by enhancing autophagy through inhibition of the PI3K/AKT/mTOR pathway. Front Pharmacol 11: 128, 2020.

26. Bu LL, Liu YQ, Shen Y, Fan Y, Yu WB, Jiang DL, Tang YL, Yang YJ, Wu P, Zuo CT, et al: Neuroprotection of exendin-4 by enhanced autophagy in a parkinsonian rat model of $\alpha$-synucleinopathy. Neurotherapeutics: Mar 15, 2021 (Epub ahead of print). 
27. Dou R, Qian J, Wu W, Zhang Y, Yuan Y, Guo M, Wei R, Yang S, Jurczyszyn A, Janz S, et al: Suppression of steroid $5 \alpha$-reductase type I promotes cellular apoptosis and autophagy via PI3K/Akt/mTOR pathway in multiple myeloma. Cell Death Dis 12: 206, 2021

28. Li J, Tian M, Hua T, Wang H, Yang M, Li W, Zhang $X$ and Yuan H: Combination of autophagy and NFE2L2/NRF2 activation as a treatment approach for neuropathic pain. Autophagy: April 9, 2021 (Epub ahead of print).

29. Shi J, Yu T, Song K, Du S, He S, Hu X, Li X, Li H, Dong S, Zhang Y, et al: Dexmedetomidine ameliorates endotoxin-induced acute lung injury in vivo and in vitro by preserving mitochondrial dynamic equilibrium through the HIF-1a/HO-1 signaling pathway. Redox Biol 41: 101954, 2021.

30. Chen J, Wang Y, Wu J, Yang J, Li M and Chen Q: The potential value of targeting ferroptosis in early brain injury after acute CNS disease. Front Mol Neurosci 13: 110, 2020.

31. Chen J, Zhang C, Yan T, Yang L, Wang Y, Shi Z, Li M and Chen Q Atorvastatin ameliorates early brain injury after subarachnoid hemorrhage via inhibition of pyroptosis and neuroinflammation. J Cell Physiol: Mar 3, 2021 (Epub ahead of print).

32. National Research Council (US) Institute for Laboratory Animal Research: Guide for the Care and Use of Laboratory Animals. National Academies Press (US), Washington, DC, 1996.

33. Flierl MA, Stahel PF, Beauchamp KM, Morgan SJ, Smith WR and Shohami E: Mouse closed head injury model induced by a weight-drop device. Nat Protoc 4: 1328-1337, 2009.

34. Tian J, Yang L, Wang P, Yang L and Fan Z: Exogenous CGRP regulates apoptosis and autophagy to alleviate traumatic brain injury through Akt/mTOR signalling pathway. Neurochem Res 45: 2926-2938, 2020.

35. Chen J, Xuan Y, Chen Y, Wu T, Chen L, Guan H, Yang S, He J, Shi D and Wang Y: Netrin-1 alleviates subarachnoid haemorrhage-induced brain injury via the PPAR gamma/NF-KB signalling pathway. J Cell Mol Med 23: 2256-2262, 2019.

36. Li G, Dong Y, Liu D, Zou Z, Hao G, Gao X, Pan P and Liang G: NEK7 coordinates rapid neuroinflammation after subarachnoid hemorrhage in mice. Front Neurol 11: 551, 2020.

37. Das S, Chattopadhyay D, Chatterjee SK, Mondal SA Majumdar SS, Mukhopadhyay S, Saha N, Velayutham R, Bhattacharya $S$ and Mukherjee S: Increase in PPAR $\gamma$ inhibitory phosphorylation by Fetuin-A through the activation of Ras-MEK-ERK pathway causes insulin resistance. Biochim Biophys Acta Mol Basis Dis 1867: 166050, 2021.

38. Zhao S, Cheng L, Shi Y, Li J, Yun Q and Yang H: MIEF2 reprograms lipid metabolism to drive progression of ovarian cancer through ROS/AKT/mTOR signaling pathway. Cell Death Dis 12: $18,2021$.

39. Dando I, Pacchiana R, Pozza ED, Cataldo I, Bruno S, Conti P, Cordani M, Grimaldi A, Butera G, Caraglia M, et al: UCP2 inhibition induces ROS/Akt/mTOR axis: Role of GAPDH nuclear translocation in genipin/everolimus anticancer synergism. Free Radic Biol Med 113: 176-189, 2017.

40. Dick RW: Is there a gender difference in concussion incidence and outcomes? Br J Sports Med 43 (Suppl 1): i46-i50, 2009.

41. Gupte R, Brooks W, Vukas R, Pierce J and Harris J: Sex differences in traumatic brain injury: What We Know and What We Should Know. J Neurotrauma 36: 3063-3091, 2019.

42. Biegon A: Considering biological sex in traumatic brain injury. Front Neurol 12: 576366, 2021.
43. Kawazoe Y, Miyamoto K, Morimoto T, Yamamoto T, Fuke A, Hashimoto A, Koami H, Beppu S, Katayama Y, Itoh M, et al: Effect of dexmedetomidine on mortality and ventilator-free days in patients requiring mechanical ventilation with sepsis: A randomized clinical trial. JAMA 317: 1321-1328, 2017.

44. Skrobik Y, Duprey MS, Hill NS and Devlin JW: Low-Dose nocturnal dexmedetomidine prevents ICU delirium. A Randomized, Placebo-Controlled Trial. Am J Respir Crit Care Med 197: 1147-1156, 2018.

45. Chen J, Wang J, Li C, Ding H, Ye J and Xia Z: Dexmedetomidine reverses MTX-induced neurotoxicity and inflammation in hippocampal HT22 cell lines via NCOA4-mediated ferritinophagy. Aging (Albany NY) 13: 6182-6193, 2021.

46. Chen X, Chen D, Li Q, Wu S, Pan J, Liao Y, Zheng X and Zeng W: Dexmedetomidine alleviates hypoxia-induced synaptic loss and cognitive impairment via inhibition of microglial NOX2 activation in the hippocampus of neonatal rats. Oxid Med Cell Longev 2021: 6643171, 2021.

47. Mei B, Li J and Zuo Z: Dexmedetomidine attenuates sepsis-associated inflammation and encephalopathy via central $\alpha 2 \mathrm{~A}$ adrenoceptor. Brain Behav Immun 91: 296-314, 2021.

48. Yang YF, Wang H, Song N, Jiang YH, Zhang J, Meng XW, Feng XM, Liu H, Peng K and Ji FH: Dexmedetomidine attenuates ischemia/reperfusion-induced myocardial inflammation and apoptosis through inhibiting endoplasmic reticulum stress signaling. J Inflamm Res 14: 1217-1233, 2021.

49. Gao Y, Zhang Y, Dong Y, Wu X and Liu H: Dexmedetomidine mediates neuroglobin Up-regulation and alleviates the hypoxia/reoxygenation injury by inhibiting neuronal apoptosis in developing rats. Front Pharmacol 11: 555532, 2020.

50. Sun YB, Zhao H, Mu DL, Zhang W, Cui J, Wu L, Alam A, Wang DX and Ma D: Dexmedetomidine inhibits astrocyte pyroptosis and subsequently protects the brain in in vitro and in vivo models of sepsis. Cell Death Dis 10: 167, 2019

51. Shen R, Pan D, Wang Z, Jin X, Li Z and Wang H: The effects of dexmedetomidine post-conditioning on cardiac and neurological outcomes after cardiac arrest and resuscitation in swine. Shock 55: 388-395, 2021.

52. Nixon RA: The role of autophagy in neurodegenerative disease. Nat Med 19: 983-997, 2013.

53. Zeng Z, Zhang Y, Jiang W, He L and Qu H: Modulation of autophagy in traumatic brain injury. J Cell Physiol 235: 1973-1985, 2020.

54. Xue H, Wu Z, Xu Y, Gao Q, Zhang Y, Li C and Zhao P: Dexmedetomidine post-conditioning ameliorates long-term neurological outcomes after neonatal hypoxic ischemia: The role of autophagy. Life Sci 270: 118980, 2021.

55. Wang M, Ke Y, Li Y, Shan Z, Mi W, Cao Y, Feng W and Zheng X: The nephroprotective effects and mechanisms of rehmapicrogenin include ROS inhibition via an oestrogen-like pathway both in vivo and in vitro. Biomed Pharmacother 138: 111305, 2021.

56. Cui G, Li Z, Cao F, Li P, Jin M, Hou S, Yang X, Mu Y, Peng C, Shao $\mathrm{H}$ and $\mathrm{Du} \mathrm{Z}$ : Activation of $\mathrm{Nrf} 2 / \mathrm{HO}-1$ signaling pathway attenuates ROS-mediated autophagy induced by silica nanoparticles in H9c2 cells. Environ Toxicol 36: 1389-1401, 2021.

This work is licensed under a Creative Commons Attribution-NonCommercial-NoDerivatives 4.0 International (CC BY-NC-ND 4.0) License. 